\title{
Frequency of Toxoplasma Infection Among Pregnant Women and Their Newborn in Kashan, Iran
}

\author{
Sima Rasti ${ }^{1, *}$; Hossein Hooshyar ${ }^{1}$; Mohsen Arbabi ${ }^{1}$; Atefeh Fatahian ${ }^{2}$; Mitra Behrashi ${ }^{2}$; \\ Ahmad Talebian ${ }^{3}$; Mojgan Bandehpour ${ }^{4}$; Seyed Gholamabbas Mousavi ${ }^{5}$ \\ ${ }_{1}^{1}$ Department of Parasitology, Kashan University of Medical Sciences, Kashan, IR Iran \\ ${ }^{2}$ Department of Obstetrics and Gynecology, Kashan University of Medical Sciences, Kashan, IR Iran \\ 3 Department of Pediatrics, Kashan University of Medical Sciences, Kashan, IR Iran \\ ${ }^{4}$ Cellular and Molecular Biology Research Center, Shahid Beheshti University of Medical Sciences, Tehran, IR Iran \\ ${ }^{5}$ Department of Statistics, Faculty of Public Health, Kashan University of Medical Sciences, Kashan,IR Iran \\ *Corresponding author: Sima Rasti, Department of Parasitology, Kashan University of Medical Sciences, Kashan, IR Iran. E-mail: rasti_s@yahoo.com
}

Received: July 5, 2014; Accepted: August 5, 2014

Background: Congenital toxoplasmosis (CT) is the result of maternal infection if acquired during gestation, an infection that most often is clinically unapparent.

Objectives: This study was conducted to determine acute and chronic toxoplamosis in pregnant women and its complications in newborn.

Patients and Methods: In this cohort study, 798 pregnant women were tested for Toxoplasma IgG/IgM by ELISA. PCR and IgM were performed on 4 newborns of acute and 5 chronic maternal infections; they were followed for 1 year. Data were analyzed by SPSS-11.5 and $\chi 2$ test.

Results: Five (0.63\%)and 341 (42.7\%) were positive for acute and chronic infections. A 400 bp band was seen in 3 newborns of acute maternal infections. The incidence of neonatal toxoplasmosis was 3.7 per 1000. Only one neonate of the acute maternal toxoplasmosis had jaundice. Conclusions: The rate of maternal and neonatal toxoplasmosis, were higher than the world. Jaundice was only sign in newborn with Toxoplasma gondii infection.

Keywords: Toxoplasmosis; Polymerase chain reaction; complications; ELISA; Pregnant women; Neonatal

\section{Background}

Congenital toxoplasmosis (CT) is the result of maternal infection if acquired during gestation, an infection that most often is clinically unapparent [1]. The prevalence of infection in the pregnant women varies from $0.79 \%$ to $84 \%$ in the world $[1,2]$. The incidence of congenital toxoplasmosis is reported, 3.3 per 10000 live births in France and 4.2 per 1000 in Iran [3, 4]. Infection in the first trimester frequently show sever congenital toxoplasmosis; stillbirth, low birth weight, hydrocephaly and retinochoroiditis $[1,5]$. Infections in the third trimester showing no sign of disease before first year of life. However, if they are left untreated, signs such as epilepsy, schizophrenia, strabismus and nonspecific illness are developed [1].

Serological test remains limited interest for diagnosis of toxoplasmosis in neonates. Detection of parasite DNA by polymerase chain reaction (PCR) is one of the best methods for diagnosis. PCR is a reliable, quick, accurate and specific method for diagnosis of infection where the immune response is abnormal or absent in fetus $[1,6]$.

\section{Objectives}

There is little information about neonatal toxoplasmosis in Iran; this study was designed to determine the prevalence of Toxoplasma gondii in pregnant women, their newborns and complications of the disease.

\section{Patients and Methods}

In this cohort study, 798 pregnant women with gestational age of more than 27 weeks admitted to ShabihKhani Maternity and Gynecology Hospital of Kashan University of Medical Sciences, Iran during Oct 2007 to Feb 2009 were studied.

Chronic maternal $T$. gondii infection diagnosed by serological test; Enzyme-linked Immunosorbent assay (ELISA); negative IgM and IgG $<1 / 400$. Acute maternal T. gondii infection diagnosed by positive IgM 1/100 and $\operatorname{IgG}>1 / 400[1]$.

PCR and ELISA/IgM were performed on four neonates were born from acute and five from chronic maternal T. gondii infection to detect neonatal toxoplasmosis.

Copyright (c) 2015, Zahedan University of Medical Sciences. This is an open-access article distributed under the terms of the Creative Commons Attribution-NonCommercial 4.0 International License (http://creativecommons.org/licenses/by-nc/4.0/) which permits copy and redistribute the material just in noncommercial usages, provided the original work is properly cited. 
Rasti S et al.

By positive IgM 1/100 or observing of $400 \mathrm{bp}$ on PCR was diagnosed as positive neonatal toxoplasmosis. One case of acute maternal T. gondii infection refused to cooperate.

Five milliliters blood samples were collected from pregnant women; isolated sera were frozen at $-20^{\circ} \mathrm{C}$ until assays. A questionnaire including demographic data, antenatal visits, clinical signs, and complications of neonatal toxoplasmosis were recorded. ELISA was used for detection of IgM and IgG antibodies to T. gondii according to the manufacturer's instructions (ADALITIS kit, Italy). Two milliliters blood of the newborns were collected in 0.5 M ethylenediamminetetraacetate (EDTA), then DNA was extracted by standard method of sodium perchlorate, PCR amplification was carried out by G529 primers (TTT TGA CTC GGG CCC AGC, GTC CAA GCC TCC GAC TCT), Taq DNA polymerase and DNA samples [6-8]. PCR program was as $94^{\circ} \mathrm{C}$ for $5 \mathrm{~min}$, followed by 30 cycles of $94^{\circ} \mathrm{C}$ for 30 seconds, $48^{\circ} \mathrm{C}$ for 30 seconds, $72^{\circ} \mathrm{C}$ for 30 seconds. PCR product was electrophoresed in $1.5 \%$ agrose gel and visualized under UV. RH strain of T. gondii used as positive control. The expected fragment size band was 400 bp, which was considered as congenital toxoplasmosis, representing, parasitemia of the newborn. Weight (kg) and gestational age (week) and growth in intrauterine by ultrasonography of four and five neonates born of acute and chronic maternal infection respectively and 28 cases of others were recorded.

The clinical signs and symptoms of the two groups of infants were followed up for one year in two consequent times by a pediatrician and ophthalmologist. If jaundice was present in face and eye of the infants, bilirubin and liver tests were recommended and performed. Total bilirubin more than $1.2 \mathrm{mg} / \mathrm{dL}$ and direct bilirubin more than $0.3 \mathrm{mg} / \mathrm{dL}$, serum glutamic oxaloacetic transaminase (SGOT) and serum glutamate pyruvate transaminase enzymes (SGPT) more than $33 \mathrm{IU} / \mathrm{L}$ and $36 \mathrm{IU} / \mathrm{L}$ were considered as liver dysfunction jaundice [9].

The data were analyzed using SPSS-11.5 (Inc. Chicago.) and outcome variables were calculated using $\chi^{2}$ test. A
$\mathrm{P}<0.05$ was considered statistically significant. This study was approved by the ethics committee of Kashan University of Medical Sciences.

\section{Results}

The seroprevalence of IgG (chronic) and IgM (acute) toxoplasmosis in pregnant women were $42.7 \%$ (341/798) and $0.63 \%$ (5/798).

Distribution of infection according to age and educational group were shown in Table 1 . High seroprevalence of IgG was seen in 30 - 39 years $62.4 \%$ ( $P<0.001)$. The prevalence of IgG in undergraduates were higher than the others $(P=0.007, O R=1.47)$ (Table 1$)$. There were no signs of fever, pain and lymphadenopathy in the pregnant women afflicted to chronic and acute infection, of which $46.9 \%$ had optic disorders. No significant relation was found between toxoplasmosis and mothers optic disorders. Out of four mothers with acute toxoplasmosis, three neonates showed a $400 \mathrm{bp}$ band in PCR. It was identified as neonatal toxoplasmosis. On the other hand, PCR was negative in the neonates was born from chronic maternal infection. The incidence rate of neonatal toxoplasmosis was 3.7 per 1000. None of the newborns were positive by ELISA/IgM.

Weight averages at time of birth in both groups were $3375 \pm 389.2 \mathrm{~g}$ and $3266 \pm 345.3 \mathrm{~g}$. Among neonates born from acute maternal infection, three neonates were born in the 39th week of pregnancy and one in 37th week. In the other group, majority of neonates were born in 3940th week. The difference was not statistically significant. One out of three patients with neonatal toxoplasmosis (33.3\%) showed jaundice, liver dysfunction. Total bilirubin, direct bilirubin, SGOT and SGPT were $6.5 \mathrm{mg}$ / dL, $3 \mathrm{mg} / \mathrm{dL}, 75 \mathrm{IU} / \mathrm{L}$ and $80 \mathrm{IU} / \mathrm{L}$, respectively, represented hyperbilirubinemia, liver dysfunction or jaundice. None of the 28 neonates born from chronic maternal infection had jaundice (Table 2). There were no sign of lymphadenopathy, neurological and ophthalmological disorders such as chorioretinitis and general symptoms among the infants of the two groups for one year.

\begin{tabular}{|c|c|c|c|c|}
\hline & \multicolumn{3}{|c|}{ IgG } & \multirow[t]{2}{*}{ P-Value } \\
\hline & Positive [N(\%)] & Negative [N(\%)] & Total (N/\%) & \\
\hline Age group, yr & & & & $<0.001$ \\
\hline$>20$ & $7(17.5)$ & $33(82.5)$ & $40(100)$ & \\
\hline $20-29$ & $209(37.7)$ & $345(62.3)$ & $554(100)$ & \\
\hline $30-39$ & $123(62.4)$ & $74(37.6)$ & $197(100)$ & \\
\hline$>40$ & $2(28.6)$ & $5(71.4)$ & $7(100)$ & \\
\hline Total & $341(42.7)$ & $457(57.3)$ & $798(100)$ & \\
\hline Educational state & & & & 0.007 \\
\hline Primary and junior school & $194(47.3)$ & $216(52.7)$ & $410(100)$ & \\
\hline Senior high school and further & $147(37.9)$ & $241(62.1)$ & $388(100)$ & \\
\hline Total & $341(42.7)$ & $457(57.3)$ & $798(100)$ & \\
\hline
\end{tabular}


Rasti S et al.

Table 2. Describing Acute Toxoplasmosis in Pregnant Women and Three Neonate's Congenital Toxplasmosis Confirmed by PCR Using Primer G529

\begin{tabular}{lccccccc}
\hline Cases & $\begin{array}{c}\text { Mother IgM } \\
\mathbf{1 / 1 0 0}\end{array}$ & $\begin{array}{c}\text { Mother IgG } \\
\mathbf{1 / 4 0 0}\end{array}$ & Neonate PCR & Neonate IgM & $\begin{array}{c}\text { Weight } \\
\text { Neonate, gr }\end{array}$ & $\begin{array}{c}\text { Gestation } \\
\text { Age, week }\end{array}$ & Jaundice \\
\hline $\mathbf{1}$ & Positive & Positive & Positive & Negative & 3600 & 39 & Negative \\
$\mathbf{2}$ & Positive & Positive & Positive & Negative & 2800 & 37 & Positive \\
$\mathbf{3}$ & Positive & Positive & Positive & Negative & 3600 & 39 & Negative \\
$\mathbf{4}$ & Positive & Positive & Negative & Negative & 3500 & 39 & Negative \\
\hline
\end{tabular}

\section{Discussion}

The results of this study showed prevalence of anti-toxoplasma IgG antibody among pregnant women was 341 (42.7\%) (95\% CI: 39.27-46.13\%). The sero-prevalence of IgG in women at childbearing age in Iran has been reported $37.6 \%$ [10]. The prevalence of IgG in Kashan's pregnant women was approximately similar as reported in France (44\%) and South America (45\%) [4,11]. This difference of infection can be related to climate condition, food habits, cultural and social situation, contact with cat and diagnostic methods $[1,4]$. In this research, there was no clinical sign among the afflicted mothers.

Toxoplasma IgG seroprevalence among women increases with age and the difference was significant, that consistence with other study [10].

The prevalence of IgG in uneducated was 1.47 fold than other groups. The relation between seroprovalence and transmission risk factors such as raw meat consumption was not significant. The incidental rate of IgM (acute toxoplasmosis) in Kashan's pregnant women was 6 per 1000 pregnancies ( 5 cases) (95\% CI: 0.05-1.15\%).

The incidence of acute maternal T. gondii infection during pregnancy varies in different countries; such as Kuwait 13.8\% [12], South America 2.8\% [11]. The incidence of acute maternal $T$. gondii infection in Kashan is less than other side of Iran [13], Kuwait and South America [11, 12], while it is more than that in Spain [14].

Three infected neonates born from acute maternal infection were positive by PCR, but no newborns in chronic infection showed positive results. The risk of transmitting infection to the fetus was $75 \%$. The rate of transmitting at 36th week of pregnancy is reported $71 \%$ [1]. This study was done in the third trimester of pregnancy in which $75 \%$ of suspected infants were diagnosed by PCR. ELISA/IgM were positive in none infants of two groups. According to findings of el Fakahany et al. IgM anti T. gondii and PCR were positive in $13 \%$ and $46.6 \%$ of babies with congenital toxoplasmosis [15], which is approximately in consistence with our findings. Infant's age, pathogenesis, number of transferred organisms, gestational age at which infection occurred and maturity of the infant's immune system play an important role in their positive serology [1]. Considering weak and positive tests, $75 \%$ of the infants afflicted with congenital toxoplasmosis do not have measurable amount of anti T. gondii IgM antibodies in their serum and may remain negative in the first year of birth. So negative serologic test does not reject the possibility of congenital infection [1].

Sensitivity of serology tests decreases two weeks after birth. In this research, infant's bleeding was performed in one month after birth which may cause pseudo-negative. Sensitivity of PCR increases with the gestational age at maternal seroconversion. The incidence rate of congenital toxoplasmosis in France is 3.3 per 10000 live births [4]. According to results of present study and Gharavi [3] neonatal toxoplasmosis were reported 3.7 and 4.2 per 1000 live births in Kashan and Tehran, Iran, that was higher than France.

The present study was performed on pregnant women at third trimester and the results showed that one of three infected neonates (33.3\%) had jaundice until one year of age, but none of the 28 infants in the other group had any complications. Majority of newborn was born from infected mothers in the third trimester have no signs of congenital toxoplasmosis but some of them had retinochoroidal after four years of age [1]. In France, by screening pregnant women, treatment and health education, seroconversion and congenital toxoplasmosis fall from $84 \%$ in 1960 s to $44 \%$ in 2003 and 1 per 1000 to 3.3 per 10000 [4]. Therefore, it is suggested that upon observing general symptoms such as jaundice in infant, attempts for detecting ophthalmologic disorder till age four and treatment have been started. In addition, health education for prevention is recommended. Results of this study showed that prevalence of IgG maternal and neonatal toxoplasmosis in Kashan, Iran, was higher than that reported in the world. PCR detects the disease in $75 \%$ of the suspected infants with negative serology. The majority of the infected infants had no signs of disease except for the jaundice until one year of age.

\section{Acknowledgements}

The study was funded by vice chancellor for research of Kashan University of Medical Sciences, Grant No: 8607. (Sima Rasti et al.). The authors are thankful to Prof. Bahram Kazemi, Dr Rahmati and Prof. Rezvan Moniri.

\section{Authors' Contributions}

Study concept, design and writing the manuscript were 
done by Sima Rasti, Mitra Behrashi, Hossein Hooshyar, Mohsen Arbabi. Atefeh Fatahian collected the data and followed clinical signs and complications the newborns Mojgan Bandepour for PCR method. Ahmad Talebian recorded complications of newborn. Data analysis was done by S. Gholamabbas Mousavi.

\section{Funding/Support}

Kashan University of Medical Sciences.

\section{References}

1. Montoya JG, Kovacs JA, Remington JS. Toxoplasma gondii. In: Mandell GL, Bennet JE, Dolin R editors. Principles and practice of infectious diseases online: PIN code and user guide to continually updated online reference. Philadelphia: Churchill Livingstone; 2010.

2. Song KJ, Shin JC, Shin HJ, Nam HW. Seroprevalence of toxoplasmosis in Korean pregnant women. Korean $J$ Parasitol. 2005;43(2):69-71.

3. Gharavi MJ. Congenital toxoplasmosis in Tehran. Kowsar Med J 2003;7(4):299-307.

4. Villena I, Ancelle T, Delmas C, Garcia P, Brezin AP, Thulliez P, et al. Congenital toxoplasmosis in France in 2007: first results from a national surveillance system. Euro Surveill. 2010;15(25)19600.

5. Freeman K, Oakley L, Pollak A, Buffolano W, Petersen E, Semprin $\mathrm{AE}$, et al. Association between congenital toxoplasmosis and preterm birth, low birthweight and small for gestational age birth BJOG. 2005;112(1):31-7.
6. Homan WL, Vercammen M, De Braekeleer J, Verschueren H. Identification of a 200- to 300 -fold repetitive 529 bp DNA fragment in Toxoplasma gondii, and its use for diagnostic and quantitative PCR. Int J Parasitol. 2000;30(1):69-75.

7. Green MR, Sambrook J. Molecular cloning: A laboratory manual.USA: Cold Spring Harbor Laboratory Press; 2012.

8. Rasti S, Behrashi M, Kazemi B, Fatahian A, Mousavi G, Namakchian M. Diagnosis of congenital toxoplasmosis by polymerase chain reaction. Indian J Med Microbiol. 2012;30(2):251.

9. Mcpherson RA, Pincus MR. Henry's clinical diagnosis and management by laboratory methods. 22th ed Philadelphia: W.B. Saunders; 2011.

10. Borna S, Shariat M, Fallahi M, Janani L. Prevalence of immunity to toxoplasmosis among Iranian childbearing age women: Systematic review and meta-analysis. Iran J Reprod Med. 2013;11(11):861-8.

11. Rosso F, Les JT, Agudelo A, Villalobos C, Chaves JA, Tunubala GA, et al. Prevalence of infection with Toxoplasma gondii among pregnant women in Cali, Colombia, South America. Am J Trop Med Hyg. 2008;78(3):504-8.

12. Iqbal J, Khalid N. Detection of acute Toxoplasma gondii infection in early pregnancy by IgG avidity and PCR analysis. J Med Microbiol. 2007;56(Pt 11):1495-9.

13. Fouladvand MA, Jafari SM. Prevalence of antibodies to toxoplasma gondii in pregnant women of Bushehr. Iran South Med J. 2001;3(2):113-6

14. Roc ML, Palacian MP, Lomba E, Monforte ML, Rebaje V, Revillo Pinilla MJ. [Serologic diagnosis of congenital toxoplasmosis]. spanish, Enferm Infecc Microbiol Clin. 2010;28(8):517-9.

15. el Fakahany AF, Abdel-Maboud AI, el-Garhy MF, Eraky MA. Com parative study between elisa IgG, IgM and PCR in diagnosing and studying toxoplasmosis in Qualyobia Governorate, Egypt.J Egypt Soc Parasitol. 2002;32(2):475-86. 